\title{
Identifying the best research design to fit the question. Part 1: quantitative designs
}

Evidence-based nursing is about applying the best available evidence to a specific clinical question. Different clinical questions require evidence from different research designs. No single design has precedence over another, rather the design chosen must fit the particular research question. ${ }^{1}$ Questions focused on the cause, prognosis (course), diagnosis, prevention, treatment, or economics of health problems are best answered using quantitative designs, whereas questions about the meaning or experience of illness are best answered using qualitative designs. Many different quantitative and qualitative research designs exist, each with a specific purpose and with strengths and limitations. In this editorial, the most rigorous quantitative designs to address questions of prevention or treatment, causation, and prognosis will be outlined. The next editorial will describe the use of qualitative designs to address questions of meaning or experience.

\section{Questions about the effectiveness of prevention and treatment interventions}

The randomised controlled trial (RCT) is the strongest design for questions of whether healthcare interventions are beneficial (ie, do more good than harm). An RCT is a true experiment in which people are randomly allocated to receive a new intervention (experimental group) or to receive a conventional intervention or no intervention at all (control group). Because it is the play of chance alone that determines the allocation, the only systematic difference between the groups should be the intervention. Investigators follow participants forward in time (follow up) and then assess whether they have experienced a specific outcome (fig 1). The 2 most important strengths of RCTs are (1) the random allocation of participants to groups, which helps to ensure that the groups are similar in all respects except exposure to the intervention, and (2) the longitudinal nature of the study, whereby exposure to the intervention precedes the development of the outcome. These 2 features ensure that any differences in outcome can be attributed to the intervention. The disadvantages of this design include the cost of conducting a trial, the long period of follow up before patients experience the outcome, and the possibility that patients who agree to participate in a trial may differ from those to whom the results would be applied (generalisability).

If you are a school nurse who wants to find an effective intervention to prevent the initiation of smoking among adolescents, you should look for evidence from RCTs. An

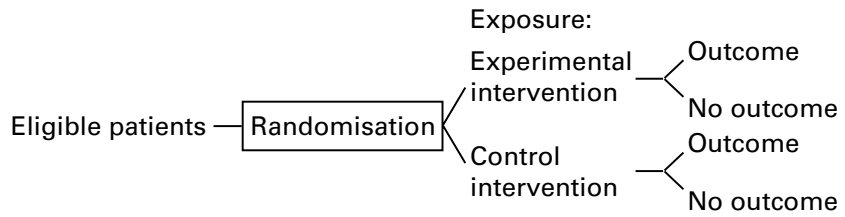

Direction of data collection: exposure $\longrightarrow$ outcome

Figure 1 Randomised controlled trial. example of a trial is one in which schools are randomly allocated to the experimental group to receive an innovative intervention which is taught in small groups and allows young adolescents the opportunity to practise smoking avoidance behaviours, whereas those allocated to the control group receive traditional lectures about the ill effects of smoking. The students are followed up for several years and data are collected and compared on the number of students in each group who begin to smoke. In most RCTs, individuals are randomly allocated to groups. In this study, schools are the unit of randomisation to reduce the likelihood of students discussing their experiences of the intervention with students in the control group. To avoid such contamination, investigators will often randomise to units such as classrooms, schools, or communities.

If you are a primary care nurse practitioner and are wondering whether you should suggest nicotine gum to help smokers to stop smoking, again you should look for evidence from RCTs. In such trials, smokers are randomly allocated to nicotine gum (experimental group) or to placebo gum, which looks and tastes like nicotine gum but contains no active ingredients (control group). They are then followed up, and data are collected and compared on the number of participants in each group who stopped smoking.

Readers of this journal will notice that all of the treatment studies that have been abstracted have been RCTs. An example from this issue is the study by Macknin (p13) on the effectiveness of zinc lozenges for cold symptoms in children. There are occasions, however, when the evaluation of an intervention using an RCT is not ethical or feasible. In this case, we must rely on a less rigorous design such as the cohort analytic study (also known as a controlled trial). This study design is similar to the RCT in that there are comparison groups who receive and do not receive an intervention and they are followed up to determine who experiences the outcome of interest. The important difference between the 2 designs is the absence of random allocation to study groups; instead, participants most often select themselves or are selected by a clinician to receive the intervention (fig 2). This is an important limitation because groups may differ in ways other than exposure to the intervention. Group differences in outcomes at the end of the study may be because of differences in the groups that existed before the intervention began (baseline differences). The intervention therefore may appear to have had an effect on the outcome when, in fact, it was the initial differences in groups that influenced the outcome.

Continuing with the example of the school based smoking prevention programme, if schools were unhappy about being randomly allocated to groups, a cohort analytic study might be done rather than an RCT. In such a study, several schools are approached and asked if they would like to receive the innovative intervention (intervention group) or the traditional lectures (control group). Without random allocation to groups, it may be that schools that choose the innovative intervention differ from those that choose not to 




Direction of data collection: exposure $\longrightarrow$ outcome

Figure 2 Cohort analytic study.

receive it in ways that may influence the outcome (eg, socioeconomic status or parental smoking habits). The study findings may show that students who received the innovative intervention were less likely to start smoking; however, this outcome may have been influenced by the baseline characteristics of the group rather than, or as well as, the intervention. In other words, this group may not have been as likely to smoke even if they did not receive the innovative intervention. Even if investigators document the group differences in baseline characteristics or use statistical techniques to adjust for the differences, other factors that were not considered may be responsible for differences in outcome.

Using a cohort analytic design for the nicotine gum example, smokers who want to stop smoking are offered nicotine gum and those who choose to take it (intervention group) are compared over time with those who choose not to take it (control group). Again, the major limitation of this design is that smokers who choose to take nicotine gum may differ from those who choose not to take it with respect to known and unknown baseline characteristics that may influence the outcome. Nicotine gum may appear to increase quit rates when, in fact, the increase may be because of variables such as higher motivation to stop smoking, younger age, or fewer years of smoking.

\section{Questions about the cause of a health problem or disease}

The RCT is the most rigorous design to determine whether some factor (exposure) causes an outcome. Using this design, participants are randomly allocated to be or not be exposed to a potential causative agent and then followed up to compare the number in each group who experience the outcome. In questions of causation, however, it may not be ethical or feasible to randomly allocate people to exposure to the causative agent. The next best evidence comes from cohort analytic studies. In this design, the investigator follows up people who are exposed to and not exposed to a causative agent. The major strength of RCTs and cohort analytic studies is that those entering the study have not yet experienced the outcome. The investigator is certain therefore that exposure to the causative agent (smoking) precedes the development of the outcome (lung cancer). This issue of temporality-that is, that the causative agent precedes the development of the outcome, is crucial for establishing a causal relation. ${ }^{2}$

When the outcome of interest is rare or takes a long time to develop, neither RCTs nor cohort analytic studies may be feasible. In these circumstances, a case control design is often used. In a case control design, patients with the outcome of interest (cases) and patients without the outcome of interest (controls) are identified and then the investigator determines whether they have had previous exposure to the causative agent (fig 3). The investigator is able to match the case and control patients on important variables that may influence the outcome (eg, age, sex, and other health conditions). In this way, the groups are as similar as possible and the specific

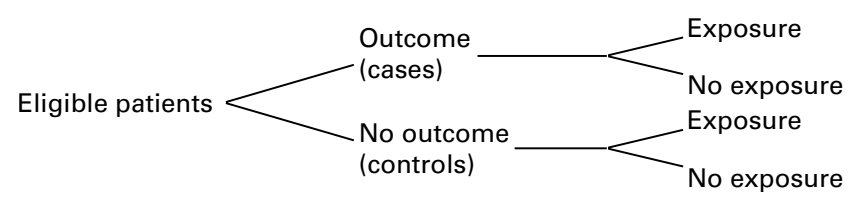

Direction of data collection: outcome $\longrightarrow$ exposure

Figure 3 Case control study.

effect of the causative agent on the outcome can be more confidently examined. The strengths of this design are that it allows the assessment of causation when the outcome is rare or takes a long time to develop, and that it includes a control group. The limitations are the difficulties in establishing that the exposure actually occurred before the outcome (temporality), obtaining accurate information about exposure to a causative agent which has occurred in the past (relies on accuracy of people's memory or on completeness and accuracy of medical records), and identifying a control group that is similar in all other factors that may have influenced the outcome.

When considering whether smoking causes lung cancer, it is unethical to randomise participants to smoke or to not smoke and then follow them up to determine who develops lung cancer; an RCT is therefore not possible. Cohort analytic studies have been done in which investigators followed up a group of smokers and a group of nonsmokers, who were matched for as many other explanatory variables as possible. However, given the long time it takes before lung cancer develops, these studies took many years to complete. Case control studies are often a more feasible option when the disease in question is rare and when a cohort analytic study would be extremely large and costly to identify a sufficient number of people who develop the disease. Using a case control design for the smoking and lung cancer example, people with lung cancer are matched, for several important variables, to people without lung cancer. All participants are asked about their past smoking behaviour, and the number of smokers in each group are compared to see whether those with lung cancer were more likely to have smoked. An example of a case control study that examines a causal relation in this issue is the study by Woodward et al (p25) on whether parenting style increases hyperactivity in school aged boys.

\section{Questions about the course of a health state or disease (prognosis)}

When we are interested in the likelihood that people will experience or develop an outcome given their exposure to a disease, condition, or situation, the best design is a cohort study. An example of a prognosis question that appeared in a previous issue of Evidence-Based Nursing is whether infants who are preterm and small for gestational age are likely to have cognitive and motor delays during early childhood (Hutton et al, Jan 1998 issue, p 19). In this example, the condition is being preterm and small for gestational age. An example of a prognosis question that begins with a disease is how likely are patients with ulcerative colitis to develop bowel cancer? In a cohort study, one group of patients who are at a uniform point in the development or exposure of the disease or condition (eg, at first onset or at initial diagnosis) (inception cohort) and free of the outcome of interest are followed up to determine who develops the outcome (fig 4).

In the case of lung cancer, an example of a prognosis study is to assemble a group of patients who have just been diagnosed with lung cancer and follow them up to determine when certain symptoms appear or how long patients survive. 


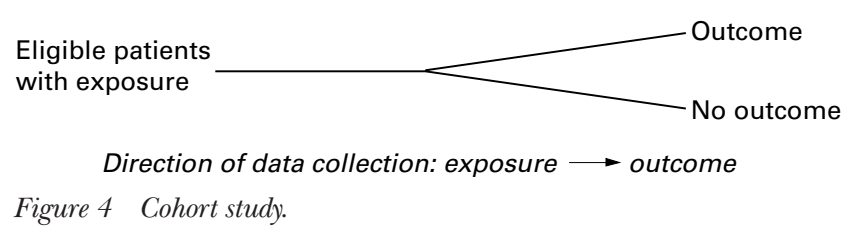

The longitudinal nature of the cohort design ensures that the disease, condition, or situation precedes the outcome. The disadvantages of this design are the expense and time required to follow up large numbers of patients until some develop the outcome.

\section{Single studies versus systematic reviews}

Questions about the prevention or treatment of disease, and the cause or course of disease are usually addressed by more than one study. To help practitioners and health policy makers keep up with the literature related to specific topics, systematic reviews of the literature are conducted. In a systematic review, eligible research studies are viewed as a population to be systematically sampled and surveyed. Individual study characteristics and results are then abstracted, quantified, coded, and assembled into a database that, if appropriate, is statistically analysed much like other quantitative data. The statistical combination of the results of $>1$ study, or meta-analysis, effectively increases the sample size and results in a more precise estimate of effect than can be obtained from any of the individual studies used in the meta-analysis. Rigorous systematic reviews provide a summary of all methodologically sound studies related to a specific topic. This is much more powerful than the results of any one single study and avoids the potential of bias if one only looks at some of the studies on a particular question. ${ }^{3}$ An example of a systematic review in this issue is the study by Tang (p18) about whether an intervention of dietary advice lowers blood cholesterol concentrations in people living in the community.

\section{Conclusion}

There are numerous quantitative research designs. The designs described above are the most rigorous for addressing questions about treatment, causation, and prognosis. When looking for studies related to these questions, awareness of these designs will help nurses to identify those studies and systematic reviews worth reading.

JACKIE ROBERTS, RN, MSc

ALBA DiCENSO, RN, PhD

School of Nursing, Faculty of Health Sciences,

McMaster University, Hamilton, Ontario, Canada

1 Mulhall A. Nursing, research, and the evidence [editorial]. Evidence-Based Nursing 1998 Jan;1:4-6.

2 Hill AB. Principles of medical statistics (9th edition). London: Lancet, 1971:313.

3 DiCenso A , Cullum N, Ciliska D. Implementing evidence-based nursing: some misconceptions [editorial]. Evidence-Based Nursing 1998 Apr;1:3840 .

\section{Implementing evidence-based leg ulcer management}

Leg ulceration is a chronic, recurring condition and it is estimated that $1 \%$ of adults will have a leg ulcer at some time. The majority of leg ulcers are due to venous disease, with arterial disease, diabetes, auto-immune diseases, and malignancy accounting for about $25 \%$. In the UK, leg ulcers are usually managed by nurses. ${ }^{1}$ A systematic review of research on compression bandaging concluded that venous ulcers should be treated with multilayer high compression bandaging. ${ }^{2}$ No particular high compression bandaging regimen was found to be superior; Unna's boot, short stretch bandages, and multilayered elastomeric systems were all effective. Simple wound dressings are generally placed underneath the bandage and over the ulcer to allow healing to occur without drying of the wound and to prevent trauma on dressing removal and sensitisation to topical preparations.

In the Wirral, a mixed rural and urban area in north west England (population 332 000), we implemented 2 effective compression bandaging regimens. The use of appropriate primary wound dressings was also implemented as there is no evidence that semiocclusive or occlusive dressings such as foams, films, or hydrocolloids are more effective than simple, inexpensive dressings (eg, knitted viscose dressings or saline gauze) for venous leg ulcers. ${ }^{3}$ This paper describes the changes in practice and patient outcomes after the implementation of evidence-based guidelines for assessment, bandaging, and wound dressing.

\section{Baseline audit}

An initial audit of leg ulcer care and patient outcomes was commissioned using data collected by local practitioners. The local prevalence of ulceration (1.42 ulcers per 1000 people) was similar to national estimates for the UK. 21 different types of dressings were used. Of 319 ulcers, 83 (26\%) were treated with dressings that contained iodine, $60(19 \%)$ with hydrocolloid dressings, and 42 (13\%) with knitted viscose dressings. 14 types of cream or ointments and 30 different types of bandages were used, of which only $21 \%$ were compression bandages. $25 \%$ of ulcers healed in 12 weeks.

In a baseline survey of knowledge and reported practice relating to leg ulcer care, nurses scored an average of $40 \%{ }^{4}$

\section{Implementing the Wirral Leg Ulcer Pilot Project}

The following were key elements required to narrow the gap between current practice and best possible practice:

- All staff in the Wirral to adopt a consistent approach to leg ulcer assessment and treatment

- Use of handheld Doppler ultrasound should be part of the assessment

- Patients should be assessed to determine suitability for compression bandages or need for referral for specialist medical opinion

- Handheld Dopplers and compression bandages should be readily available

- Nurses should be trained in the application of compression bandages 
- Nurses should apply a simple low adherent dressing to venous leg ulcers.

Although it was clear that multilayer compression was beneficial, the best method of application was unclear. It was decided to advocate the use of 3 and 4 layer systems (table). This minimised costs while enabling nurses and patients to exercise choice.

Compression therapies used in the Wirral

\begin{tabular}{|c|c|c|}
\hline & 4 layer system & 3 layer system \\
\hline 1st layer & Orthopaedic wool padding & Orthopaedic wool padding \\
\hline 2nd layer & Crepe bandage* & $\begin{array}{l}\text { High power, highly } \\
\text { extensible, compression } \\
\text { bandage* }\end{array}$ \\
\hline 3rd layer & $\begin{array}{l}\text { Low power, highly } \\
\text { extensible, elastomeric } \\
\text { compression bandage }\end{array}$ & $\begin{array}{l}\text { Shaped tubular elastomeric } \\
\text { bandage }\end{array}$ \\
\hline 4th layer & $\begin{array}{l}\text { Medium power, moderately } \\
\text { extensible, cohesive } \\
\text { bandage }\end{array}$ & \\
\hline
\end{tabular}

*Available on prescription. The 2nd and 3rd layers of both systems are washable and reusable, but in practice the 4 layer system was used once and discarded.

The estimated cost of using each of the two bandaging regimens for 12 weeks was $£ 103.08$ for the 4 layer, and between $£ 49.22$ and $£ 100.32$ for the 3 layer regimen (1996 costs), depending on the ability and propensity of patients to wash and reuse their bandages.

\section{SECURING FUNDING FOR THE PROJECT}

First year costs (setup, bandages, and evaluation) were $£ 135000$ and recurrent annual costs are $£ 55000$ (to purchase the elements not available on prescription). The local health authority, which purchases health care for local residents, agreed to provide $£ 70000$ for the first year to buy treatments not available on prescription (such as orthopaedic wool, shaped tubular bandages, and cohesive bandages) and to fund a nurse specialist. The development, implementation, and evaluation of the nurse training package cost an additional $£ 39000$.

\section{NURSE TRAINING}

The nurse training programme comprised:

- 2 study days with lectures on leg ulcer aetiology, assessment, treatment, prevention of recurrence, referral, and skin care

- Practical workshops on using handheld Dopplers and applying compression bandages

- A video showing application of the 4 layer and 3 layer bandaging regimens

- Visits to leg ulcer clinics to observe and practise patient assessment and bandage application

- A training package containing reference information and self assessment exercises.

\section{DEVELOPMENT OF A DATABASE}

The introduction of the guideline was identified as an opportunity to set up a system to monitor the process and patient outcomes in leg ulcer management. The local community care providers developed a comprehensive database, which enabled ongoing audit of patients with venous leg ulcers. Nurses sent 3 monthly updates on all patients with venous leg ulcers to the clinical nurse specialist and these data were used to generate reports on patient outcomes.

\section{Outcomes}

USE OF COMPRESSION BANDAGING

Between July 1995 and April 1997, 138 patients (36\%) were treated with 3 layer bandaging and 194 (51\%) with 4 layer bandaging (ie, a total of $87 \%$ received compression compared with $21 \%$ at baseline). 45 (12\%) did not tolerate high compression as per the guideline and were treated using reduced compression, 2 received no bandaging, and 1 person wore a compression sock.

\section{USE OF PRIMARY DRESSINGS}

At baseline, the most commonly used primary dressings were hydrocolloid sheets (19\%), simple dressings (14\%), and iodine impregnated petroleum gauze (14\%). After introduction of the guideline, fewer than $5 \%$ of patients were treated with a hydrocolloid, and 94\% were treated with a simple non-adherent dressing. The range of dressing types decreased from 21 before implementation to 5 after implementation. There is no evidence that dressings that contain iodine or hydrocolloid dressings are more effective than simple dressings, ${ }^{3}$ and hence the cost difference (approximately $£ 2.00$ compared with $£ 0.30$ per dressing) may result in considerable savings.

NURSE TRAINING

There were significant changes in knowledge about leg ulcer assessment, treatment, and reported practice after nurses completed the training programme. ${ }^{4}$ Bandaging skill also improved: before training, most nurses applied compression bandages in such a way as to produce a tourniquet effect. After training this fell to $40 \%$ of nurses for the 4 layer and $64 \%$ of nurses for the first 2 layers of the 3 layer regimen. ${ }^{4}$

\section{ULCER HEALING RATES}

Patient and ulcer healing rates were assessed quarterly using the audit data. Some patients had $>1$ ulcer; presenting the healing rates by patient gives a lower figure because patient healing requires that all of a patient's ulcers heal. Although this study did not evaluate the bandages per se, and patients were not randomised to the alternatives, we compared the proportion of patients whose ulcers completely healed using the different systems; these were not significantly different (34\% v 32\% at $12 \mathrm{wk}$ and $60 \%$ \% 54\% at $24 \mathrm{wk}$, in the 3 and 4 layer systems, respectively). The healing rate for patients treated with reduced compression therapy was 19\% at 24 weeks. One of the barriers to documenting the results was that nurses failed to report when healing occurred.

\section{Barriers to implementation}

\section{ORGANISATION AND FUNDING OF SERVICES}

Thorne conducted a systematic review of community leg ulcer clinics and found that although they appear to improve patient outcomes, poor research design meant that it was not possible to tell whether improvements in outcome were independent of other changes in the service, such as provision of training or bandaging. ${ }^{5}$ Given that Cullum and Last found that $78 \%$ of patients receiving district nurse visits for leg ulcer treatment were fully mobile, it seemed sensible to establish clinics so that nurse travel time could be minimised. ${ }^{6}$ Initially, plans were drawn up for weekly community leg ulcer clinics in each of the 6 areas of the Wirral. Some general practitioners (GPs), however, especially those who employed nurses directly to work with their caseloads, refused to allow "their" staff to treat other patients. A compromise was reached whereby patients received care in one of 3 settings-community clinics in health centres where patients of many GPs were seen, clinics in GP premises where patients from that practice were seen, and at home.

\section{HOSPITAL SECTOR}

Although most leg ulcers can be treated in the community, recalcitrant leg ulcers require referral to hospital for further investigation and treatment. A few patients with uncomplicated leg ulcers may be treated in hospital if they are admit- 
ted with co-existing medical problems. Unfortunately, few hospital nurses were able to attend the training. This threatened the continuity of care between hospital and community. The community leg ulcer specialist was frequently called into the hospital to treat patients because no nurses were available who had undergone training in the application of compression bandages.

Within the local secondary care setting, only the 4 layer bandage system was used because of the consultant's preferences and because a component of the 3 layer regimen (class $3 \mathrm{c}$ bandage) was not readily available through the hospital stores. This posed a problem for patients who had already started the 3 layer regimen in the community and had to change to the 4 layer if they were referred to secondary care.

LACK OF AVAILABILITY OF BANDAGE COMPONENTS

Some components of the effective bandaging systems (eg, orthopaedic wool padding, shaped tubular bandages, and cohesive bandages) were not available on prescription. This project was possible because the health authority provided $£ 55000$ each year for the purchase of non-prescription items. Thus the cost of setting up a similar service in other districts may be prohibitive until the issue of provision of items not available on prescription is addressed.

\section{Lessons learnt}

- The provision of research funds to develop, implement, and evaluate a comprehensive training package was crucial to the implementation and success of the project

- Involvement of the independent sector (ie, nursing homes) was essential to ensure continuity of care

- Involvement of the hospital tissue viability nurse and senior nursing or clinic managers might have increased the uptake of the training by hospital nurses and reduced the need for the project nurse to treat patients in the hospital

- Multilayer compression bandaging was effective in settings other than leg ulcer clinics

- Even after training not every nurse could apply compression therapy to the same standard

- 3 layer compression therapy can be more difficult to apply than 4 layer, but the comparability in terms of cost effectiveness is unclear.

\section{Conclusions}

We reported the improvements in practice and patient outcomes after the implementation of an evidence-based guideline. The approach to implementation was multifaceted, incorporating interactive study days, audit and feedback, paper-based teaching materials and guidelines, video, educational outreach, and organisational change. In the evaluation, we could neither determine the relative contribution of the different implementation strategies nor of the individual changes to leg ulcer care such as change in bandage use, nurse training, and reorganisation of service. Implementation of the guideline, however, was clearly associated with improved practice and patient outcomes.

ANGELA TINKLER, BSc(HONS), PG DIP JULIE HOTCHKISS, SRN, MPH, HON MFPHM Wirral Health Authority

St Catherine's Hospital,

Merseyside, UK

University of York,

E ANDREA NELSON, RGN, BSc(HONS)

York, $U K$

(Department of Nursing, University of Liverpool at time of project)

LIZ EDWARDS, RGN, DN, BSc(HONS) Wirral and West Cheshire Community NHS Trust,

Merseyside, UK

We are grateful to North West Region NHS Executive for funding the project.

1 Fletcher A. The epidemiology of leg ulcers. In Cullum N, Roe B, editors. Leg ulcers: nursing management. London: Balliere Tindall, 1996.

2 Fletcher A, Cullum N, Sheldon TA. A systematic review of compression treatment for venous leg ulcers. BMJ 1997;315:576-80.

3 Bradley M, Nelson EA, Cullum N, et al. A systematic review of dressings and topical agents in chronic wounds. NHS health technology assessment programme report, in press.

4 Jones JE, Nelson EA. Evaluation of an education package in leg ulcer management. J Wound Care 1997;6:342-3.

5 Thorne E. Community leg ulcer clinics and the effectiveness of care. $J$ Wound Care 1998;7:94-9.

6 Cullum N, Last S. The prevalence, characteristics, and management of leg ulcers in a UK community. In: Harding KG, Cherry GW, Dealey C, et al, editors. Proceedings of 2 nd European conference on advances in wound management. London: Macmillan Magazines, 1993.

\section{Invitation to submit letters and papers on implementation}

\footnotetext{
$A^{\text {n }}$ $\mathrm{n}$ important step in the practice of evidence-based nursing is the sharing of successful implementation strategies. We therefore welAcome submission of manuscripts describing the process and results of the implementation of an evidence-based nursing intervention. Manuscripts should be no longer than 1500 words, including references. All manuscripts will be peer reviewed and submission does not guarantee publication.

We also welcome letters from our readers about Evidence-Based Nursing. We would like to hear about the positive and negative aspects of our journal. Your feedback is most important in assisting us to produce a high quality journal which is useful to the practising nurse. Letters should be no longer than 400 words.

All submitted material should be typewritten, double spaced, and mailed or faxed to Nicky Cullum in the UK editorial office (Centre for Evidence Based Nursing, University of York, Genesis 6, York Science Park, York YO10 5DQ. Fax: + 44 (0) 1904434102 ).
}

\section{Publication dates of Evidence-Based Nursing in 1999 \\ - January • April • July • October}

